\title{
Impactos de uma pandemia na saúde mental: analisando o efeito causado pelo COVID-19
}

The impacts of a pandemic on the mental health: analysing the effect caused by COVID19

Los impactos de una pandemia en la salud mental: analizando el efecto causado por el COVID-19

Sílvia Eutrópio Vasconcelos ${ }^{1 *}$, Paula Eduarda Barcelos Dias ${ }^{1}$, Heluma Kiister Bitencourt', João Pedro Satuf Silva de Carvalho', Erika de Almeida Santos Quadros', Mariana Melo Franco Viviani', Ana Laura Horta Nunes', Carlos Eduardo Resende Sampaio'.

\section{RESUMO}

Objetivo: Investigar as implicações na saúde mental em decorrência de surtos e de pandemias, com enfoque na COVID-19. Revisão bibliográfica: A presença atual da COVID-19, em adição de outras pandemias/epidemias anteriores ou outros eventos de acometimento global, é uma importante causa de impactos negativos para a saúde mental da população, desencadeando ou agravando sintomas de ansiedade, depressão, estresse, medo, e até alguns transtornos, como transtorno de estresse pós traumático (TEPT), entre outros. Estes sintomas são frequentemente relatados por trabalhadores de serviços essenciais - profissionais da área da saúde, porteiros, caixas de supermercado etc - indivíduos acometidos pela doença e seus familiares. Além disso, a instituição de medidas de isolamento como forma de prevenção às doenças em tempos de pandemia impacta diretamente na saúde mental da população como um todo. Pouco tem sido relatado, contudo, sobre o assunto. Considerações finais: A literatura é clara no que se refere aos impactos na saúde mental de toda a população durante uma pandemia, sendo capaz de repercutir na qualidade de vida tanto individualmente quanto coletivamente.

Palavras-chave: Saúde mental, Pandemia, Epidemia.

\begin{abstract}
Objective: To investigate the implications on mental health due to outbreaks and pandemics, focusing on Covid-19. Bibliographic review: The current presence of Covid-19, in addition to other previous pandemics/epidemics or other globally affected events, is an important cause of negative impacts on the population's mental health, triggering or aggravating symptoms of anxiety, depression, stress, fear, and even some mental disorders, such as post-traumatic stress disorder (PTSD), among others. These symptoms are often reported by essential service workers - healthcare professionals, doormen, supermarket cashiers, etc. individuals affected by the disease and their families. In addition, the settling of isolation measures as a way of preventing diseases in times of pandemic directly impacts on the mental health of the population as a whole. Little has been reported, however, on the subject. Final considerations: The literature is unanimous regarding that the pandemic causes a negative reaction on the mental health of the entire population, capable to have an adverse effect on the quality of life both individually and collectively.
\end{abstract}

Keywords: Mental health, Pandemics, Epidemics.

${ }^{1}$ Pontifícia Universidade Católica de Minas Gerais, Betim - MG.

*E-mail: sileutropio@gmail.com

SUBMETIDO EM: 8/2020

ACEITO EM: 9/2020

PUBLICADO EM: 12/2020 


\section{RESUMEN}

Objetivo: Investigar las implicaciones en la salud mental debido a brotes y a pandemias, con enfoque en la COVID-19. Revisión bibliográfica: La presencia actual de la COVID-19, juntamente a otras pandemias/epidemias anteriores $u$ otros eventos de acometimiento global, es una importante causa de impactos negativos para la salud mental de la población, desencadenando o agravando síntomas de ansiedad, depresión, estrés, miedo, y hasta mismo algunos trastornos, como trastorno de estrés postraumático (TEPT), entre otros. Estes síntomas son frecuentemente relatados por trabajadores de servicios esenciales - profesionales de la salud, porteros, cajas de supermercados etc - individuos con la enfermedad y sus familiares. Además de eso, la institución de medidas de aislamiento como forma de prevención a las enfermedades en tiempos de pandemia impacta directamente en la salud mental de la población en general. Todavía, poco es relatado sobre en asunto. Consideraciones finales: La literatura es clara cuando se refiere a los impactos en la salud mental de la población general en una pandemia, capaz de repercutir en la calidad de vida tanto individualmente cuanto colectivamente.

Palabras clave: Salud mental, Pandemia, Epidemias.

\section{INTRODUÇÃO}

Segundo Santos EG e Siqueira MM (2010), cerca de 20 a 35\% da população brasileira possui algum tipo de transtorno mental, com ênfase nas profissões julgadas como essenciais como trabalhadores rurais, enfermeiros, ACS, motoristas e cobradores. Isso se deve a rotina de trabalho, a demanda, a exigência, o controle, o processo de trabalho e as condições ambientais; fatores que são piorados em um contexto de pandemia. Os transtornos mais prevalentes na população brasileira são os transtornos da ansiedade, transtornos do humor e os transtornos relacionados ao uso de substâncias psicoativas (SANTOS EG e SIQUEIRA MM, 2010).

Sociedades acometidas por episódios de desastres em larga escala, como doenças pandêmicas globais, desastres naturais, tragédias provocadas pelo homem, conflitos de guerra, crises sociais, etc., podem ser acometidas por traumas emocionais profundos e desordens relacionadas ao estresse, como transtornos do humor e ansiedade. Cerca de $16 \%$ da população global é afetada por distúrbios de saúde mental e esse número tende a aumentar com a presença de eventos de escala global, como pandemias. No ano de 2020 a presença da COVID-19 é mais um exemplo de um fator negativo na saúde mental das pessoas, exacerbando sintomas de ansiedade, depressão, solidão, estresse e medo. (COSIC K, et al., 2020; WANG J, et al., 2020).

O COVID-19 foi identificado pela primeira vez em 01 de dezembro de 2019 na cidade de Wuhan, China como um quadro de pneumonia desconhecida e, devido a sua alta capacidade de proliferação, o acesso a viagens internacionais e até mesmo a facilidade das pessoas de se locomover de um lugar para outro, a Organização Mundial da Saúde (OMS) declarou estado de pandemia mundial no dia 11 de março de 2020 (MACEDO J e ADRIANO M, 2020). Até o dia 01 de agosto, foram confirmados 17.580.163 casos de COVID19 no mundo; sendo Estados Unidos o país com o maior número de casos acumulados (4.562.037), seguido pelo Brasil (2.707.877), Índia (1.695.988) e Rússia (839.981) e África do Sul (493.183) (BRASIL, 2020). Em relação aos óbitos, foram confirmados 679.501 no mundo até o dia 18 de julho; sendo Estados Unidos o país com maior número acumulado de óbitos (153.314), seguido do Brasil (93.563), México (46.688), Reino Unido (46.119) e Índia (36.511) (BRASIL, 2020). O coeficiente de incidência bruto no mundo ao final do mês de julho foi de 2.255 casos para cada 1 milhão de habitantes (BRASIL, 2020).

Segundo Zhang Y e Ma ZF (2020), apesar de estudos recentes publicados na revista The Lancet abordarem a prevenção da disseminação da COVID-19 e os sintomas clínicos dos pacientes infectados, muito pouco tem sido relatado sobre o impacto na qualidade de vida e na saúde mental durante a pandemia da COVID-19, mesmo com o grave acometimento da doença na China e em outras partes do mundo. A fim de melhor compreender os impactos na saúde mental de desastres em larga escala, é possível, contudo, utilizar a epidemia de Síndrome Respiratória Aguda Grave (SARS) em 2003 como um evento que permita a melhor 
compreensão da epidemia do COVID-19, tendo em vista a escassez de bibliografias sobre o assunto. Estimase que a SARS (2003), que foi controlada em oito meses, tenha causado 800 mortes em todo o mundo (26 países acometidos) (ZHANG Y e MA ZF, 2020).

Ademais, foram relatados nas áreas severamente afetadas pela epidemia de SARS sintomas de estresse pós-traumático moderados a graves. Ainda segundo Zhang Y e Ma ZF (2020), em outras epidemias, como a H1N1, Síndrome respiratória do Oriente Médio (MERS) e Ebola, os impactos incluíram transtornos por uso de substâncias e depressão.

Somado a isso, alguns fatores de risco conhecidos para depressão e ansiedade foram evidenciados como agravantes do impacto negativo à saúde mental que as epidemias provocam. (Alguns exemplos de fatores agravantes são a alta taxa de mortalidade da doença, uma possível insegurança alimentar e de recursos, discriminação e contato com indivíduos infectados e doentes) (ZHANG Y e MA ZF, 2020)

Esse estudo tem como objetivo investigar as implicações na saúde mental em decorrência de surtos e de pandemias, trazendo para o centro da análise a COVID-19 (devido a sua contemporaneidade). Além disso, o estudo busca ampliar o olhar sobre o sofrimento psíquico e relatar a importância de se pensar na saúde mental e no bem-estar das pessoas submetidas a esse período de crise em saúde pública.

\section{REVISÃO BIBLIOGRÁFICA}

O novo coronavírus (COVID-19), caracterizado como pandemia pela OMS em março de 2020, tem criado reflexos para as organizações governamentais, economias e principalmente, para a população. Ao traçar um paralelo com registros de impactos à saúde mental em outras epidemias e situações de conflito pode-se inferir que as consequências para a saúde mental da população provavelmente serão significantes.

Cosic K, et al. (2020) chama atenção para a sobrecarga do sistema de saúde mental, já insuficiente em muitos dos mais de 200 países afetados pelo novo coronavírus (DEPIERRO J, et al., 2020; ZHANG Y e MA ZF, 2020; COSIC K, et al., 2020).

\section{Impacto nos trabalhadores de serviços essenciais}

O atual cenário de sobrecarga dos sistemas de saúde faz com que seja necessário que os serviços se organizem de forma a atender o maior número de pessoas possível dependendo do menor uso de insumos. Dessa forma, os trabalhadores da área da saúde são desafiados a gerenciar esses insumos durante sua atuação nos diversos centros de cuidado.

Segundo Depierro J, et al. (2020) e Marcinko D, et al. (2020), os profissionais de saúde, principalmente aqueles que se encontram na linha de frente ao combate do COVID-19, estão susceptíveis ao desenvolvimento de ansiedade e transtornos afetivos, principalmente por carregarem responsabilidades diante da saúde de seus pacientes.

Esses profissionais são encarregados diariamente de fazer escolhas para o racionamento de recursos (como a transferência entre unidades de tratamento maior complexidade) e insumos hospitalares (como ventiladores) dentro das unidades de tratamento, essas escolhas são decisivas para o desfecho que 0 paciente terá. Greenberg N, et al. (2020) afirma que diante desse cenário, é possível que algumas escolhas acarretem a transgressão de estatutos morais causando a chamada injúria moral.

A ocorrência dessa está associada ao profundo sentimento de culpa, vergonha e descontentamento, além de maior risco para distúrbios psiquiátricos e ideação suicida. Grande ênfase é dada ao possível desenvolvimento de TEPT, depressao e uso abusivo de substâncias (DEPIERRO J, et al., 2020; GREENBERG N, et al., 2020; MARCINKO D, et al., 2020).

O impacto na saúde mental de trabalhadores não se restringe ao campo da saúde. Profissionais de várias categorias como entregadores de comida, policiais, equipes de limpeza e funcionários de supermercados continuaram a trabalhar in loco enquanto advogados, administradores e consultores migraram para o trabalho remoto em casa. 
$\mathrm{Na}$ impossibilidade de se manter em isolamento social, esses trabalhadores fazem parte de serviços que não podem ser interrompidos. Trabalhadores de serviços essenciais não-médicos podem ter maior risco de desenvolver doenças psiquiátricas crônicas em comparação aos profissionais da saúde. Isso pode ser inferido ao traçar um paralelo da atual pandemia do COVID-19 com o atentado terrorista do dia 11 de Setembro de 2001 nos Estados Unidos. Nesse episódio, socorristas voluntários, profissionais da construção civil e trabalhadores de asbesto tiveram maior ocorrência de TEPT do que profissionais como bombeiros e policiais. Isso provavelmente ocorre pois o primeiro grupo recebeu menos apoio social, não possuía experiência em resposta a desastres e se viu assumindo tarefas não usuais, geralmente não por opção, mas devido à necessidade econômica (DEPIERRO J, et al., 2020).

\section{Impacto nos infectados pelo vírus e seus familiares}

Os pacientes com suspeita ou com confirmação de COVID-19 apresentam sintomas de excitação, impulsividade e agressividade após serem informados de sua condição. Eles podem não cooperar no tratamento, negar sua doença ou até mesmo se recusar a serem isolados.

Estão mais susceptíveis à quadros como ansiedade, depressão, negação e insônia, o que pode ser explicado pela incerteza de sua condição de saúde e medo de possíveis complicações ou agravamento do quadro, podendo interferir no tratamento e até mesmo elevar o risco para agressões e suicídio e também para momentos de pânico (LI W, et al., 2020; WANG J, et al., 2020).

Somado a isso, para evitar a infecção cruzada, os pacientes infectados geralmente são admitidos em alas separadas e isoladas e também submetidos a um período prolongado de quarentena após a alta. Estando sozinhos por um período extenso, esses pacientes podem sofrer de solidão. Ainda nessas condições a equipe médica responsável pelos cuidados usa roupas grossas de isolamento, de modo que os sentimentos de intimidade e confiança que um paciente deve sentir ao estar em contato com a equipe são substituídos pelo medo.

O número crescente de infecções e mortes por COVID-19 também pode aumentar o receio desses pacientes. Por último, mas não menos relevante, existe a questão sobre o uso de glicocorticóides no tratamento, que também pode causar alterações de humor, excitação, agitação, falta de sono, aumento de fala e movimentos, irritabilidade e outros sintomas.

Em relação a discordâncias no uso dos medicamentos Cloroquina e Hidroxicloroquina que tem sido o centro de muitos debates no tratamento da doença, a recomendação da Organização Mundial de Saúde é a de que os medicamentos não têm comprovação científica de que sejam seguros e eficazes no tratamento da COVID-19, incluindo alertas de sérios efeitos colaterais, como labilidade emocional, nervosismo e até mesmo comportamentos suicidas. Nesse sentido, como ainda não existe um medicamento ou uma vacina específicos para a doença, as pessoas podem criar maiores medos e ansiedade por não estarem recebendo tratamento de fato eficaz (WANG J, et al., 2020).

As famílias de pacientes falecidos durante a pandemia podem sofrer sensação de perda e luto. Além disso, outro fator que impacta diretamente na saúde mental dessas pessoas em períodos de pandemia, principalmente nesse momento de COVID-19, é a impossibilidade de vivência desse luto de maneira convencional. Por exemplo, familiares e amigos não podem, na maioria das vezes, participar dos enterros (AFONSO P, 2020; LI W, et al., 2020).

Nos mais de 200 países afetados pela pandemia não existe estrutura e profissionais suficientes para o aumento significativo da demanda de serviços de saúde mental que está por vir. Como exemplo, o número de psiquiatras por 100.000 habitantes na China é 2,2; já no Reino Unido, 11 e segundo a OMS este número no Brasil em 2016 era de 3,165, enquanto o recomendado em 2008 para grandes cidades era de 10 psiquiatras para 100.000 habitantes, de acordo com a Revised Framework for Psychiatry.

Esse aumento da demanda num sistema de cuidados em saúde mental já sobrecarregado apresenta um risco de gerar uma crise de saúde mental por todo o mundo, o que seria extremamente maléfico para os pacientes e suas famílias e frustrante para os que trabalham na área (COSIC K, et al., 2020; DEPIERRO J, et al., 2020). 


\section{Os efeitos da quarentena}

Como forma de manejo e enfrentamento da pandemia do novo coronavírus os órgãos da saúde de países afetados optaram por instituição da quarentena. A quarentena é a retirada de pessoas sadias do convívio social como forma de evitar o contágio pelo vírus; o isolamento é relevante para a proteção da saúde física populacional, contudo, o cenário de quarentena, além de aumentar os riscos de sofrimento por doenças psiquiátricas, também impacta o meio social de várias outras formas (AFONSO P, 2020).

Traumas emocionais profundos são intensificados ou criados em larga escala por desastres humanos como doenças pandêmicas, desastres naturais, conflitos de guerra e outros. Com foco no surto de doenças infecciosas, situação que se enquadra no cenário atual, a população sofre um abalo psicológico por causa de diversos fatores, o que pode fragilizar a saúde mental. Nesses períodos, há divulgação excessiva ou inadequada de informações nos veículos de comunicação como televisão, jornais e internet e isso pode contribuir para que as pessoas tenham uma afinidade maior por informações negativas e informais, gerando também a diminuição da confiabilidade de informações positivas e formais.

Soma-se a essa conjuntura a presença de especulações e rumores que podem causar apreensão nas pessoas por não saberem ao certo os reflexos de uma situação atípica como essa tanto no contexto pessoal quanto no contexto nacional. As autoridades tomando medidas de emergência, como bloquear cidades, instituir fechamento de serviços como bares, restaurantes, shoppings, também é outro ponto a ser destacado que pode impactar diretamente a vida social, já que os encontros, confraternizações e formas de se relacionar com outras pessoas ficam afetadas.

Como consequência dessas ações, a economia pode sofrer por diminuição do comércio e pela urgência do remodelamento das formas de consumo, além de haver desespero por suprimentos e consequentemente, a falta deles em supermercados e farmácias. O fato da doença ser ostensivamente falada e divulgada, até por pôsteres nas ruas, podem deixar as pessoas nervosas e assustadas quando resolvem sair de casa (WANG J, et al., 2020).

Estudos apontam que a repercussão mais significativa desse momento será a elevação de taxas de estresse, ansiedade, depressão e TEPT. Isso especialmente se liga aos efeitos da quarentena nas atividades diárias, nas rotinas e meios de subsistência da população. As pessoas estão apreensivas; muitos não sabem lidar com o cenário do isolamento e podem recorrer a formas não saudáveis de fazê-lo, além de se sentirem despreparadas e desamparadas para enfrentar momentos atípicos como esse.

Dessa maneira, Cosic K, et al. (2020) também afirma que, durante esse período, já se é esperado um aumento dos níveis de solidão, depressão, uso abusivo de drogas e álcool, automutilação, comportamentos suicidas e isolamento social exagerado. Como consequência, é possível dizer que a pandemia revela ser um grande problema para saúde mental mundial (COSIC K, et al., 2020).

Como forma de ilustrar as consequências da pandemia do COVID-19 na saúde mental, foi realizada uma pesquisa com 5000 chineses. Como resultado, 21.5\% dos entrevistados registraram sintomas de TEPT, o que se assemelha as porcentagens dos sintomas de TEPT e depressão avaliados durante uma pesquisa realizada durante o surto de SARS em 2003. Além disso, em uma pesquisa feita com 2,091 habitantes da China, a prevalência de TEPT era de $4.6 \%$, sendo que nas cidades onde havia um maior número de contaminados pelo COVID-19, a prevalência demonstrou ser $18.4 \%$.

Já nos Estados Unidos, uma pesquisa realizada em março de 2020 demonstrou que $32 \%$ dos adultos puderam perceber que o estresse e a preocupação relacionados à pandemia tinham repercussão negativa na saúde mental própria; $14 \%$ ainda relataram que a situação era o impacto negativo mais significativo em relação à saúde mental (COSIC K, et al., 2020; HAMEL L, et al., 2020; HAWRYLUCK L, et al., 2004; KIRTON D, 2020; SUN L, et al., 2020).

Como já foi citado anteriormente, a escassez de profissionais especialistas em saúde mental e a sobrecarga dos serviços se mostra inapta para realizar tratamento das pessoas necessitadas durante 0 período da pandemia. 
Na realidade atual, há ainda vários empecilhos para o tratamento de doenças mentais no geral e é possível dizer que as consequências da falta de tratamento ou tratamento inadequado dos transtornos mentais são vastas.

Essa situação, além de afetar os indivíduos e famílias, também afeta a sociedade com a perda da produtividade de trabalho, desemprego, problemas familiares, violência doméstica, abuso de álcool e drogas e suicídios (COSIC K, et al., 2020).

\section{Prevenção aos impactos na saúde mental durante a pandemia do COVID-19}

Visando a preservação da saúde mental da população, é necessário que seja feito um mapeamento da população visando seus fatores de risco para desencadear repercussões psiquiátricas durante tempos de pandemia.

Essa estratificação de risco é importante pois possibilita a realização de intervenções de forma mais precoce e eficaz. Além disso, é necessário que os profissionais atuantes na atenção básica e no pronto socorro estejam atentos e aptos a identificar doenças psiquiátricas (HO C, et al., 2020).

Para preservar sua saúde mental, os profissionais de serviços essenciais devem procurar realizar atividades de autocuidado, realizar pausas durante o trabalho e evitar a exposição excessiva aos conteúdos midiáticos. Em relação aos trabalhadores da saúde, é essencial que haja suporte emocional dentro das instituições que mantêm as unidades de cuidado.

Isso pode ser feito através de turnos menores, pausas programadas para descanso durante o trabalho, incentivo a pedir ajuda, comunicação clara entre supervisores e companheiros de trabalho e treinamento para a prevenção de contaminação. Além disso, as instituições devem ter protocolos claros e precisos disseminados entre os profissionais (GREENBERG N, et al., 2020; HO C, et al., 2020).

A população, enquanto enfrenta um momento cheio de incertezas e medo durante isolamento social deve ser encorajada a buscar ajuda em serviços de atenção à saúde mental. Muitos profissionais da saúde mental se disponibilizaram para realizar atendimento gratuitos a pessoas diretamente afetadas pela pandemia. Atualmente isso é facilitado pela possibilidade de videoconferências através da internet (DEPIERRO J, et al., 2020).

A pandemia causada pelo novo coronavírus impactou a sociedade como um todo e como apresentado anteriormente, os autores deste artigo consideram que esse impacto foi enorme na saúde mental, não apenas de profissionais da saúde ou de pessoas contaminadas pelo vírus, mas de toda a população mundial.

A principal causa de impacto considerada pelos autores e confirmada pelos estudos apresentados foi a necessidade de manter o isolamento social por períodos prolongados de tempo, uma vez que nós somos seres sociais e não estamos mais acostumados a ficarmos limitados ou sem contato com outros indivíduos.

Essa questão do isolamento social ainda nos traz questionamentos sobre privilégios e condições socioeconômicas, pois fazer de fato o isolamento implica para a maior parte da população uma redução de renda e de empregos. Dessa forma, é imprescindível que os governos formem uma base sólida de combate à pandemia, não apenas incentivando sua população ao isolamento, mas proporcionando um cenário oportuno para que isso seja de fato possível.

\section{CONSIDERAÇÕES FINAIS}

As consequências da pandemia, individuais e coletivas, são desastrosas para a saúde mental, independente da preexistência ou não de distúrbios psiquiátricos, agravando ou desencadeando sintomas destes. Dessa forma, o estudo é pertinente ao analisar essas consequências, porém, possui limitações pela pandemia do COVID-19 ser recente, não havendo muitas informações disponíveis e carecendo de estudos longitudinal. Assim foi necessário comparar desfechos ocorridos em pandemias passadas com os do cenário atual, evidenciando a importância de novos estudos para fomentar a discussão acerca do tema para o desenvolvimento de estratégias que combatam o adoecimento mental da população inserida nessa realidade. 


\section{REFERÊNCIAS}

1. AFONSO P. O Impacto da Pandemia COVID-19 na Saúde Mental. Acta Med Port, 2020; 33(5):351-358.

2. BRASIL. Ministério da Saúde. Secretaria de Vigilância à Saúde. Boletim epidemiológico especial: Doença pelo Coronavírus COVID-19. Brasília, DF, 2020.

3. COSIC K, et al. Impact of human disasters and Covid-19 pandemic on mental health: potencial of digital psychiatry. Psychiatria Danubina, 2020; 32(1):25-31.

4. DEPIERRO J, et al. Lessons learned from 9/11: Mental health perspectives on the COVID-19 pandemic. Psychiatry Res, 2020; 288:113024.

5. GREENBERG N, et al. Managing mental health challenges faced by healthcare workers during covid-19 pandemic. BMJ, 2020; 368:m1211.

6. HAMEL L, et al. KFF coronavirus poll: March 2020. 2020. In: KFF. Disponível em https://www.kff.org/global-healthpolicy/poll-finding/kffcoronavirus-poll-march-2020/

7. HAWRYLUCK L, et al. SARS control and psychological effects of quarantine. Emerg Infect Dis, 2004; 10:1206

8. HO C, et al. Mental health strategies to combat the psychological impact of COVID-19 beyond paranoia and panic. Ann Acad Med Singap, 2020; 49(3):155-60.

9. KIRTON D. Chinese public dial in for support as coronavirus takes mental toll. 2020. In: Reuters. Disponível em: https://www.reuters.com/article/us-china-health-mental/chinese-public-dial-in-for-support-as-coronavirus-takesmental-toll-idUSKBN2070H2

10. LI W, et al. Progression of Mental Health Services during the COVID-19 Outbreak in China. Int J Biol Sci, 2020; 16(10): $1732-1738$.

11. MACEDO J, ADRIANO M. Covid-19: calamidade pública. Cognitionis publishing, 2020; 1-6.

12. MARCINKO D, et al. The Importance Of Psychodynamic Approach During Covid-19 Pandemic. Psychiatria Danubina, 2020; 32(1):15-21.

13. SANTOS EG, SIQUEIRA MM. Prevalência dos transtornos mentais na população adulta brasileira: uma revisão sistemática de 1997 a 2009. Jornal Brasileiro de Psiquiatria, 2010; 59(3): 238-246.

14. SCHEFFLER RM, et al. Mental Health In Central And Eastern Europe: Improving Care And Reducing Stigma Important Cases For Global Study. Londres: World Scientific, 2020; 268p.

15. SOUZA LPS, et al. Prevalência de transtornos mentais comuns em adultos no contexto da Atenção Primária à Saúde. Revista Portuguesa de Enfermagem de Saúde Mental, 2017; 1(18):1.

16. SUN L, et al. Prevalence and risk factors of acute posttraumatic stress symptoms during the COVID-19 outbreak in Wuhan, China. MedRxiv, 2020;

17. WANG J. The Psychological Impact of COVID-19 on Chinese Individuals. Yonsei Med J, 2020; 61(5):438-440.

18. WHO. Director-General's opening remarks at the media briefing on COVID-19 - 11 March 2020. 2020. In: WORLD HEALTH ORGANIZATION. Disponível em: https://www.who.int/dg/speeches/detail/who-director-general-s-openingremarks-at-the-media-briefing-on-covid-19---11-march-2020.

19. WHO. Global Health Observatory data repository: Data by country, Psychiatrists working in mental health sector (per 100000 population). 2016. In: WORLD HEALTH ORGANIZATION. Disponível em: https://apps.who.int/gho/data/node.main.MHHR?lang=en.

20. ZHANG Y, MA ZF. Impact of the COVID-19 Pandemic on Mental Health and Quality of Life among Local Residents in Liaoning Province, China: A Cross-Sectional Study. Int J Environ Res Public Health, 2020;17(7): 2381. 